\title{
Cognitive consequences of metabolic disorders
}

\author{
Urszula Łopuszańska ${ }^{1, A, C-F} \oplus$, Krystian Cholewa ${ }^{2, B} \oplus$, Magdalena Kozyra ${ }^{2, B} \odot$ \\ ${ }^{1}$ Department of Applied Psychology, Medical University, Lublin, Poland \\ 2 Students' Science Club of the Department of Applied Psychology, Medical University, Lublin, Poland \\ A - Research concept and design, B - Collection and/or assembly of data, C - Data analysis and interpretation, \\ $D$ - Writing the article, E - Critical revision of the article, F - Final approval of article
}

\begin{abstract}
Łopuszańska U, Cholewa K, Kozyra M. Cognitive consequences of metabolic disorders. J Pre-Clin Clin Res. 2020; 14(4): 139-144.
\end{abstract} doi: $10.26444 / j p c c r / 129264$

\section{Abstract}

Introduction and objective. Metabolic disorders are associated with a number of negative health consequences. Therefore, the aim of this review was to define the relationships between overweight and obesity, lipid metabolism disorders, hypertension, disorders of carbohydrate metabolism, and cognitive functions.

Abbreviated description of the state of knowledge. Metabolic disorders may be linked to the reduction of cognitive functions, such as memory, abstract reasoning, verbal fluency, attention and psychomotor speed. Obesity may lead to changes in the hippocampus, one of the main structures involved in learning and memory. The evidence reviewed suggests that obesity, by inducing inflammation, leads to the dysfunction of brain structures such as the frontal cortex and corpus callosum involved in plasticity processes and cognitive functions. In addition, obesity has been found to be associated with abnormalities in the white matter and glial cells in the brain, which may also cause reduction in processing speed. It is possible that there is a non-linear relationship between lipids and cognitive function moderated by age and the proinflammatory process. Disturbances in carbohydrate metabolism can also lead to cognitive impairment, such as: memory, verbal fluency or abstract reasoning.

Conclusions. Metabolic disorders are associated with a higher risk of developing cognitive impairment. This association can be moderated by additional factors: lifestyle, oxidative stress, pro-inflammatory processes, genetic factors, age and education.

\section{Key words}

obesity, cognitive functions, hypertension, lipids, overweight

\section{INTRODUCTION}

Metabolism is a term used to describe all biological transformation of energy in the body to sustain life. Metabolism is influenced by hormones, genetic factors and lifestyle, including diet. Irregularities in metabolism lead to a number of disorders [1]. The most important risk factors leading to metabolism disorders are overweight and obesity, both affecting undeveloped and developed countries. These factors bear the hallmarks of an epidemic that has not been effectively controlled by modern health care systems [2]. In more than 70 countries, the prevalence of obesity has doubled since 1980 and is still increaing [3].

Overweight may lead to a number of chronic negative health consequences, such as insulin resistance, type 2 diabetes, dyslipidaemia, hypertension, metabolic syndrome, atherosclerosis and cardiovascular diseases (CVDs) [4]. Metabolic disorders increase the risk of mortality in both obese adults and adults with normal body mass index (BMI) [5]. Excessive body weight is also associated with negative psychological consequences that contribute to a deterioration in the quality of life, self-esteem, and eating attitudes. Persons with overweight and obesity are more likely to suffer from symptoms of depression and anxiety [6]. Obesity is often associated with social stigma, so that persons with obesity are not socially accepted because they are regarded as not being motivated and inefficient [7].

Apart from the emotional effects of overweight and obesity, a significant problem seems to be the link between

Address for correspondence: Urszula Łopuszańska, Department of Applied Psychology, Medical University, Chodzki 15, 20-093, Lublin, Poland

E-mail: urszula.lopuszanska@umlub.pl

Received: 16.10.2020; accepted: 29.10.2020; first published: 09.11.2020 excess body weight and other disorders, such as lipid and carbohydrate metabolism disorders, as well as cognitive disorders [8]. Current studies emphasize that metabolic disorders can disturb cognitive performance, such as attention and executive function [9].

\section{OBJECTIVE}

The aim of this descriptive review was to study the link between metabolic disorders and cognitive functions in adults. Most of the research and analyzes conducted so far has focused on selected metabolic disorders, e.g. obesity and its impact on cognitive performance. We wanted to try to merge the findings about how chronic disturbed metabolic management affects brain changes that leads to cognitive disorders. We analyzed the influence of particular metabolic disorders, i.e. overweight and obesity, lipids metabolism disturbances, carbohydrate metabolism disorders, hypertension and metabolic syndrome in the development of cognitive disorders. Our attention has been focused mainly on research using brain imaging methods in humans and in animal studies.

\section{MATERIALS AND METHOD}

The PubMed/MEDLINE databases were searched for articles published between 2010-2020 describing the psychological issues of metabolic disorders, using the key words: 'overweight and obesity and cognitive function', 'lipid metabolism and cognitive function', 'carbohydrate metabolism and cognitive function' 'hypertension and cognitive function', 'metabolic 
syndrome and cognitive function'. A total of 6,919 articles were analyzed, and the analysis excluded those in which the persons examined had a diagnosis of mental disorders and mental disabilities. Finally, the analysis included 64 articles, particularly those published after 2012, as well as those in which techniques of neuro-imaging of the brain and neuropsychological methods were applied.

\section{DESCRIPTION OF THE STATE OF KNOWLEDGE}

Overweight and obesity and cognitive functions. Obesity leads to a reduction in cognitive functions, particularly in the area of memory, reasoning, motor control [10-13]. Clinical evidence shows that obesity correlates with an increased risk of developing CVDs and dementia, including Alzheimer's disease (AD) and vascular dementia (VaD), both linked to a decline in cognitive functions. This relationship was observed in the 37-years of study by Gustafson et al. The analysis of BMI in a group of 1,462 women showed that overweight and obesity, especially in middle-aged women, may increase the risk of dementia [14]. High BMI in the elderly, however, did not increase the risk of dementia. Furthermore, correlation between both overweight and obesity in middle-aged individuals and late-onset dementia, $\mathrm{AD}$, and $\mathrm{VaD}$ has been demonstrated [15].

As stated above, it has been proposed by Stanek et al. that the link between increased BMI and reduced cognitive functions derives from the deterioration of white matter and glial cells in the brain, leading to reduced neural transmission and reduced speed of processing information. In fact, a negative correlation between the BMI and the integrity of brain structures, such as the corpus callosum, has been detected. This correlation increased with the age of the participants of the study [11].

Obesity may additionally lead to chronic inflammatory conditions characterized by higher levels of $\mathrm{C}$ - reactive protein (CRP), fibrinogen and amyloid $A$ in the blood. These factors may be a source of vascular diseases because they contribute to the formation of atherosclerotic plaques [16]. Studies using magnetic resonance imaging (MRI) showed that a high concentration of CRP in blood was accompanied by dysfunction of the frontal cortex and corpus callosum, reduced executive functions, and decreased psychomotor speed and attention [17].

Roy et al., investigating the link between increased weight and reduced cognitive functions, observed changes in the PPAR a receptor (a peroxisome proliferator-activated receptor alpha), responsible for the control of the metabolism of fat in the liver in rodents. They found that an increase in body mass leads to a reduction in the level of PPARa in the whole body, including brain structures such as the hippocampus, a structure crucial for learning and memory. The PPARa receptor in the hippocampus controls calcium influx and the expression of different brain plasticity genes, such as the transcription factor protein cyclic AMP response elementbinding protein (CREB). It was observed that mice with a reduced level of PPAR $\alpha$ receptor in the hippocampus had worse spatial memory and learning ability in comparison to control mice with a normal level of PPARa. Furthermore, the injection of PPAR a into the hippocampus of the animals induced significant improvements in their learning and memory abilities [18]. The exact mechanisms connecting body weight and cognitive functions are not fully defined and require further investigation.
Accounting for genetic markers of obesity may be helpful in explaining the relationship between obesity and cognitive impairment. Vainik et al., based on the modern belief that most obesity-related genes are expressed in the central nervous system, performed studies to investigate whether neuro-behavioural factors were associated with the hereditary variability of obesity as measured by BMI. They showed that increased BMI was associated with disturbances in visualspatial functions, episodic verbal memory, impulsivity and flexibility. Elevated BMI was associated with a decrease in cortical thickness in the right frontal lob, and an increase in thickness in the left frontal lobe, especially in the lateral prefrontal cortex. These changes are called the 'right brain hypothesis'. The authors postulate that obesity is inherited through brain function [19].

Lipids metabolism disturbances and cognitive function. Lipid disorders contribute to the development of atherosclerosis, which leads to the development of CVDs [20]. Insulin cerebral circulation relates to a decline in cognitive functions due to occurred neuropsychological dysfunctions. Previous studies investigating metabolism disturbances and cognitive functions indicated that increased concentration of cholesterol in blood was associated with a reduction in mental speed, psychomotor performance, visuospatial abilities, short-term memory, operating memor, and deferred memory, as well as verbal fluency [21-23]. A balanced cholesterol concentration seems to be important as there are studies showing that low concentrations of total cholesterol are also related with both reduced cognitive functions, and these relationships are related to age. Carrington et al. showed that people over the age of 70 achieved the best results in the cognitive test (logical memory) with higher and lower levels of total cholesterol than average (U-shaped). In contrast, people under the age of 70 achieved worse cognitive performance with higher and lower levels of total cholesterol than the average (inverted-U-shape) [24].

As mentioned earlier, lipid disorders are not only linked with $\mathrm{VaD}$ but also with AD [25]. AD is characterized by the presence of amyloid plaques mainly containing aggregated forms of the beta-amyloid (Ab) peptides in the brain. In relation to molecular mechanisms of the disease, it has been shown that a high level of cholesterol, particularly the LDL fraction, increases the activity of beta-secretase enzymes that catalyze the reaction of formation of $\mathrm{Ab}$.

Liu et al., searching for lipid biomarkers characteristic of $\mathrm{VaD}$, showed that most of the lipids belonging to the classes of ceramides (Cer), cholesterol esters (ChE) and phospholipids, were significantly lower in the plasma of people with $\mathrm{VaD}$, while glycerides were elevated compared to those without $\mathrm{VaD}$. The reduced concentration of Cer and $\mathrm{ChE}$ was associated with a reduction in attention, processing speed and executive functions. The observed differences in lipid levels were not associated with changes in white matter hyperintensity and diffusion tensor imaging (DTI) measures [26]. The effect of lipid metabolism on cognitive functions requires further study because the lipid abnormalities shown in some scientific studies indicate an opposite relationship to that described above. For example, Henderson et al. produced evidence suggesting that increased levels of LDL-C were associated with increased memory abilities in middle-age women [27]. It is emphasized that among lipids, a high concentration of triglycerides (TG) is associated with the 
deterioration of cognitive functions and the occurrence of mild cognitive impairment (MCI) and AD [28].

The contemporary view may explain the contradictory results of research regarding the influence of disturbed lipid metabolism on cognitive functions, which indicates that it is not only high levels of LDL-cholesterol, triglycerides or dyslipidemia, that can lead to vascular changes. It is widely believed that serum cholesterol and lipoproteins affect the endothelium, leading, inter alia, to atherosclerotic lesions. According to Tsoupras et al., it is not high cholesterol that affects the endothelium, but the inflammatory response that moderated endothelial integrity and functionality. Longterm inflammation may contribute to an increased risk of atherosclerosis, as well as affecting the concentration and function of serum lipids, leading to the oxidation of LDL by reducing the ability of HDL to prevent LDL oxidation [29].

Bojar et al. showed that the carrier of the APOE gene polymorphism was an important factor modulating cognitive functions. It was shown that the presence $\varepsilon 3 / \varepsilon 4$, or $\varepsilon 4 / \varepsilon 4$ polymorphisms were related with worse cognitive functions, while $\varepsilon 2 / \varepsilon 3$ polymorphism of APOE gene impacted positively on cognitive functions in postmenopausal women [30]. In another study, Bojar et al. found that the carrier of the APOE gene polymorphism was an important factor modulating the relationship between lipid concentrations and the efficiency of cognitive functions. In postmenopausal women, the carrier state of the APOE gene polymorphism was associated with triglyceride levels and the results in three cognitive functions: executive functions, psychomotor speed, and cognitive flexibility. Interestingly, weight loss in postmenopausal women was associated with lower scores in cognitive tests. Cognitive performance in postmenopausal women was not significantly associated with the lipid profile [31].

Other researchers point out that a high-calorie diet induces oxidative stress, and eating large amounts of fat causes an overproduction of circulating free fatty acids and systemic inflammation. Immune cells, free fatty acids, and circulating cytokines reach the hypothalamus and initiate local inflammation through processes such as microglia proliferation [32].

Disturbances of carbohydrate metabolism and cognitive functions. When considering the correlation between metabolic disorders and cognitive functions, particular attention should be paid to the effect of glucose on the brain. Despite the fact that the human brain is only about $2 \%$ of the total body weight, it consumes approximately $75 \%$ of the glucose available, and is therefore the most metabolically demanding human organ [33]. Disturbances in the metabolism of glucose affect the efficiency of cognitive functions, as evidenced by the numerous studies carried out in type 1 and type 2 diabetic patients [34-39]. Kerti et al. showed that in people with pre-diabetes a lower level of blood glucose in comparison to the control group was associated with better cognitive efficiency in terms of cognitive functions, such as learning and memory abilities. Neuro-imaging of the brain also showed that people with higher levels of glucose in the blood were characterized by a reduced hippocampus volume [40]. Cognitive impairment in diabetes parallels a progressive vascular pathology, and increasing permeability of the blood-brain barrier (BBB) [41]. A reduction in cognitive performance induced by diabetes, as well as other metabolic disorders, constitutes a risk factor for
AD [42]. Gudala et al. showed that diabetes mellutis increased the risk of dementia by $73 \%$, a $56 \%$ increase in $\mathrm{AD}$, and a $127 \%$ increase in $\mathrm{VaD}$ [43].

Disturbances of carbohydrate metabolism can also lead to cognitive impairment, such as abstract reasoning, information processing, memory, attention, verbal fluency and the ability to learn, which deteriorates with age [42-45]. Diabetes is a chronic disease and cognitive functions are required for daily life activity. A deficiency in these cognitive abilities directly affects the health status and contributes to the irregularity in diabetes treatment [46].

Hypertension and its impacts on cognitive functions. Overweight and obesity lead to the emergence of a number of negative consequences, which include, inter alia, hypertension. Although this is undoubtedly related with decrease in the efficiency of cognitive functions, on the other hand, hypertension may be the result of emerging metabolic disorders $[47,48]$. The risk factors for hypertension include genetic factors, diet and socio-economic conditions [18]. Additionally, stress and coping abilities seem to play a crucial role [49]; for example, the influence of social factors such as social support on arterial hypertension has been well described. Acute and chronic stress lead to the development of a long-term inflammatory process which, consequently, contributes to the creation of atherosclerosis and the deterioration of cognitive functions $[50,51]$.

A study by Uiterwijk's et al. has shown that in patients with arterial hypertension there occurs a higher risk of disease of the small vessels and neuropsychological complications involving memory and speed of processing information. The analysis showed that the subjective feeling of reduced cognitive functions in patients with essential hypertension correlated positively with objective neuropsychological examination and MRI results [52].

Long-term hypertension can impair the microcirculation of the brain, degenerate the central cerebral vessels, reduce perfusion, and induce the stenosis of blood vessels. Hypertension also leads to changes in the hippocampus, also affected in AD [53], and some studies suggest that hypertension can be a risk factor for the development of AD $[54,55]$. Undoubtedly, long-term hypertension due to damages in the vascular and cardiovascular systems, leads to reduced cognitive efficiency, particularly executive functions, language, attention, processing speed and memory [56].

Metabolic syndrome and cognitive functions. The metabolic syndrome (MetS) is a group of related metabolic disorders which constitute risk factors of CVDs, diabetes and stroke. There are several definitions of MetS, but all emphasize the coexistence of abdominal obesity, impaired glucose tolerance, hypertriglyceriaemia, and hypertension. According to the International Diabetes Federation (IDF), MetS criteria are abdominal obesity (population specific) in addition to two of the following risk factor - blood glucose $(\geq 100 \mathrm{mg} / \mathrm{dL}$ (includes diabetes), dyslipidaemia (TG $\geq 1.69 \mathrm{mmol} / \mathrm{L}$, or on TG treatment; HDL-C: men $<1.03 \mathrm{mmol} / \mathrm{L}$, women $<1.29 \mathrm{mmol} / \mathrm{L}$ Or HDL treatment), blood pressure ( $\geq 130 / 85$ $\mathrm{mmHg}$ or on anti-hypertensive medicationand obesity [57].

Yates et al. demonstrated the negative impact of MetS on cognitive functions, a relationship observed even in studies in which the influence of the socio-economic and psychological factors, such as depression, were controlled. MetS was 


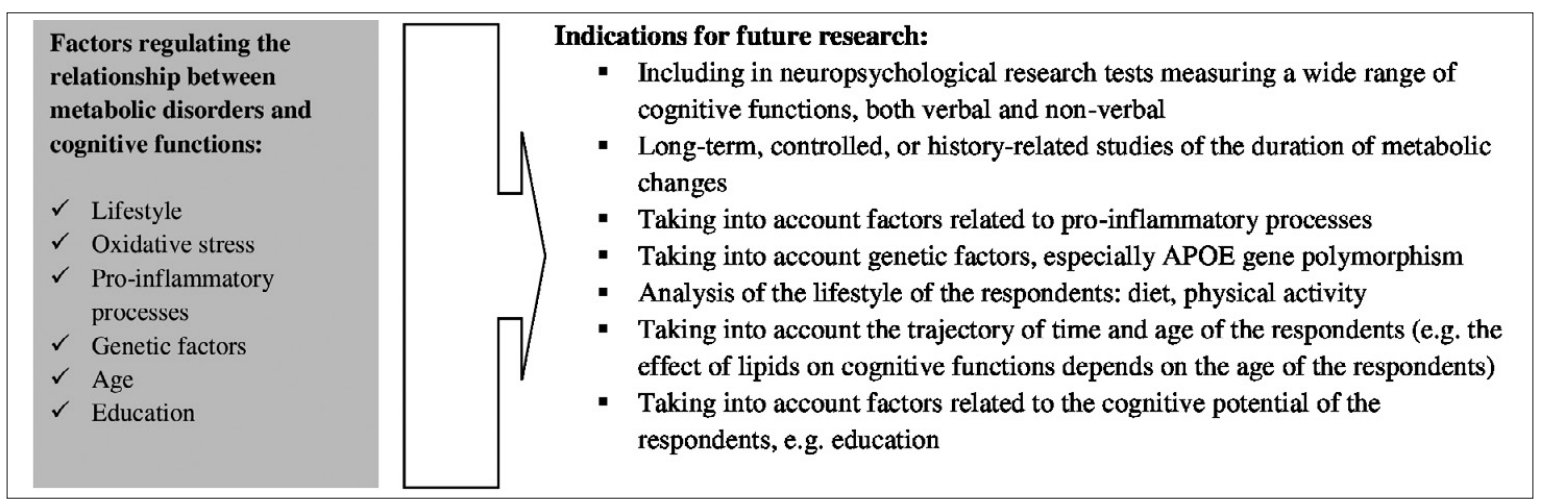

Figure 1. Recommendations for planning future research on the relationship between metabolic disorders and cognitive functions

associated with deficits in memory, visuospatial and executive abilities, speed processing and general cognitive abilities [58]. In another study, 819 patients without stroke events or a diagnosis of dementia were analyzed for the impact of MetS on cognitive function using MRI. MetS was diagnosed in $28.3 \%$. Patients with MetS, scored worse in tests of cognitive functions (memory and executive functions) than the control group. Interestingly, this correlation was found only in men. The study using MRI shows no significant differences that would explain the cognitive dysfunction in patients with MetS [59]. Exalto et al. conducted research in a group of 86 patients (mean age: 66 years) of which $41 \%$ showed a diagnosis of MetS. A neuropsychological diagnosis and brain imaging using MRI did not show significant differences in cognitive abilities, severity of vascular disorders and MRI parameters, and $\mathrm{Ab}$ levels in the cerebral spinal fluid (CSF) between patients with and without MetS. However, the occurrence of MetS was associated with the deterioration of executive functions, memory and speed processing, as well as with the deterioration of visuo-constructive abilities [60].

The Northern Manhattan Study (NOMAS) by Levin's et al. in group of 1,290 persons from urban areas and without ischemic episodes showed that considering all the signs of MetS, hypertension was the strongest predictor of cognitive deficits. According to the authors, hypertension may be a marker for neurodegenerative changes [61].

Both the persistent high concentrations of blood glucose and cholesterol, as well as untreated high blood pressure, may lead to CVDs and neuropsychological dysfunctions. Yaffe et al., during a 25 year research programme, investigated 3,000 participants for their level of cognitive abilities, blood pressure, plasma glucose, and cholesterol levels. The results clearly showed that individuals who at baseline had increased metabolic values over time, developed cognitive impairments such as information processing and memory deficits [62].

\section{CONCLUSIONS}

Cognitive functions ensure efficient functioning of the individual, and are essential for the proper functioning of the individual when deciding about the quality of life, professional success and a healthy lifestyle. Metabolic disorders contribute to the decline in cognitive functions and these relationships are enhanced with age. In the first phase, the cognitive disorders can lead to cognitive deterioration as well to MCI, or even progress to dementia [63].
Figure 1 shows both the factors regulating the relationship between metabolic disturbances and cognition, and recommendations for future research in this area. The relationship between metabolic disorders and cognitive functions may be moderated by inflammatory processes and the carrier of the APOE gene polymorphism. It seems that not only the increased parameters of metabolic disorders, such as increased body weight, abdominal obesity, increased blood lipids and glucose level, directly cause cognitive impairment. Their chronic nature seems to be of key importance, as it is associated with a long-lasting inflammatory process leading to changes in the brain, and thus cognitive changes.

Metabolic disorders can be modified by lifestyle, which seems to play a crucial role in treatment. Bajerska et al. analyzed the dietary habits in a group of 87 persons over the age of 60 , and conducted neuropsychological examination in the range of visual memory, executive functions, concentration and general cognitive abilities. The consumption of full-fat dairy products, meat products, especially red meat, was associated with decreased executive function and general cognitive abilities. In addition, insufficient physical activity, low educational status, female gender, and life without children?, (relationships without children? childless marriages?) increased the risk of cognitive deficits [64].

\section{REFERENCES}

1. Cornejo MP, Hentges ST, Maliqueo M, et al. Neuroendocrine Regulation of Metabolism. J Neuroendocrinol. 2016; 28(7): 10.1111/jne.12395. https://doi.org/10.1111/jne.12395

2. Hruby A, Hu FB. The Epidemiology of Obesity: A Big Picture. Pharmacoeconomics. 2015; 33(7): 673-689. https://doi.org/10.1007/ s40273-014-0243-x

3. Afshin A, Forouzanfar MH, Reitsma MB, et al. Health Effects of Overweight and Obesity in 195 Countries over 25 Years. N Engl J Med. 2017; 6;377(1): 13-27. https://doi.org/10.1056/NEJMoa1614362

4. Mellendijk L, Wiesmann M, Kiliaan AJ. Impact of Nutrition on Cerebral Circulation and Cognition in the Metabolic Syndrome. Nutrients. 2015; 7(11): 9416-39. https://doi.org/10.3390/nu7115477

5. Shi TH, Wang B, Natarajan S. The Influence of Metabolic Syndrome in Predicting Mortality Risk Among US Adults: Importance of Metabolic Syndrome Even in Adults With Normal Weight. Prev Chronic Dis. 2020; 17: 200020. http://dx.doi.org/10.5888/pcd17.200020

6. Değirmenci T, Kalkan-Oğuzhanoğlu N, Sözeri-Varma G, Özdel O, Fenkçi S. Psychological Symptoms in Obesity and Related Factors. Noro Psikiyatr Ars. 2015; 52(1): 42-46. https://doi.org/10.5152/npa.2015.6904

7. Fulton M, Srinivasan VN. Obesity, Stigma And Discrimination. In: StatPearls [Internet]. Treasure Island (FL): StatPearls Publishing. 2020. https://www.ncbi.nlm.nih.gov/books/NBK554571/, (access 10.10.2020). 8. Anstey KJ, Cherbuin N, Budge M, et al. Body mass index in midlife and late-life as a risk factor for dementia: a meta-analysis of prospective 
studies. Obes Rev. 2011; 12(5): 426-37. https://doi.org/10.1111/j.1467 4789X.2010.00825.X

9. Guicciardi M, Crisafulli A, Doneddu A, Fadda D, Lecis R. Effects of Metabolic Syndrome on Cognitive Performance of Adults During Exercise. Front Psychol. 2019; 10: 1845. https://doi.org/10.3389/ fpsyg.2019.01845

10. Gunstad J, Strain G, Devlin J, et al. Improved memory function 12 weeks after bariatric surgery. Surg Obes Relat Dis. 2011; 7(4): 465-472. https://doi.org/10.1016/j.soard.2010.09.015

11. Stanek KM, Grieve SM, Brickman AM, et al. Obesity is associated with reduced white matter integrity in otherwise healthy adults. Obesity (Silver Spring). 2011; 19(3): 500-4. https://doi.org/10.1038/oby.2010.312

12. Hou Q, Guan Y, Yu W, et al. Associations between obesity and cognitive impairment in the Chinese elderly: an observational study. Clin Interv Aging. 2019; 14: 367-373, https://doi.org/10.2147/CIA.S192050

13. Cheng J, East P, Blanco E, et al. Obesity leads to declines in motor skills across childhood. Child Care Health Dev. 2016; 42(3): 343-350. https:// doi.org/10.1111/cch.12336

14. Gustafson DR, Bäckman K, Joas E, et al. 37 years of body mass index and dementia: observations from the prospective population study of women in Gothenburg, Sweden. J Alzheimers Dis. 2012; 28(1): 163-71. https://doi.org/10.3233/JAD-2011-110917

15. Anjum I, Fayyaz M, Wajid A, et al. Does Obesity Increase the Risk of Dementia: A Literature Review. Cureus. 2018; 10(5): e2660. https://doi. org/10.7759/cureus. 2660

16. Ellulu MS, Patimah I, Khaza'ai H, et al. Obesity and inflammation: the linking mechanism and the complications. Arch Med Sci. 2017; 13(4): 851-863. https://doi.org/10.5114/aoms.2016.58928

17. Wersching H, Duning T, Lohmann H, et al. Serum C-reactive protein is linked to cerebral microstructural integrity and cognitive function. Neurology. 2010; 74(13): 1022-9. https://doi.org/10.1212/ WNL.0b013e3181d7b45b

18. Roy A, Jana M, Corbett GT, et al. Regulation of CREB and hippocampal plasticity-related genes by peroxisome proliferator-activated receptor $\alpha$ Cell Rep. 2013; 4(4): 724-737. https://doi.org/10.1016/j.celrep.2013.07.028

19. Vainik U, Baker TE, Dadar M, et al. Neurobehavioral correlates of obesity are largely heritable. Proc Natl Acad Sci USA. 2018; 115(37): 9312-9317. https://doi.org/10.1073/pnas.1718206115

20. Linton MRF, Yancey PG, Davies SS, et al. The Role of Lipids and Lipoproteins in Atherosclerosis. [Updated 2019 Jan 3]. In: Feingold KR, Anawalt B, Boyce A, et al, editors. Endotext [Internet]. South Dartmouth (MA): MDText.com, Inc.; 2000, https://www.ncbi.nlm.nih. gov/books/NBK343489 (access 18.09.2020).

21. Kraal AZ, Moll AC, Arvanitis NR, et al. Metabolic syndrome is negatively associated with cognition among endothelial nitric oxide synthase (eNOS)- 786C carriers in schizophrenia-spectrum disorders. J Psychiat Res. 2019; 117: 142-147. https://doi.org/10.1016/j.jpsychires.2019.07.006

22. Ancelin ML, Ripoche E, Dupuy AM, et al. Gender-specific associations between lipids and cognitive decline in the elderly. Eur Neuropsychopharmacol. 2014; 24(7): 1056-66. https://doi.org/10.1016/j. euroneuro.2014.02.003

23. He Q, Li Q, Zhao J, et al. Relationship between plasma lipids and mild cognitive impairment in the elderly Chinese: a case-control study. Lipids Health Dis. 2016; 15(1): 146. https://doi.org/10.1186/s12944-016-0320-6

24. Wendell CR, Zonderman AB, Katzel LI, et al. Nonlinear associations between plasma cholesterol levels and neuropsychological function. Neuropsychology. 2016; 30(8): 980-987. https://doi.org/10.1037/ neu0000298

25. Liu Y, Chan DKY, Thalamuthu A, et al. Plasma lipidomic biomarker analysis reveals distinct lipid changes in vascular dementia. Comput Struct Biotechnol J. 2020; 18: 1613-1624. https://doi.org/10.1016/j. csbj.2020.06.001

26. Liu Y, Chan DKY, Thalamuthu A, et al. Plasma lipidomic biomarker analysis reveals distinct lipid changes in vascular dementia. Comput Struct Biotechnol J. 2020; 18: 1613-1624. https://doi.org/10.1016/j. csbj.2020.06.001

27. Leritz EC, McGlinchey RE, Salat DH, et al. Elevated levels of serum cholesterol are associated with better performance on tasks of episodic memory. Metab Brain Dis. 2016; 31(2): 465-473. https://doi.org/10.1007/ s11011-016-9797-y

28. Morley JE, Banks WA. Lipids and cognition. J Alzheimers Dis. 2010; 20(3): 737-47. https://doi.org/10.3233/JAD-2010-091576

29. Tsoupras A, Lordan R, Zabetakis I. Inflammation, not Cholesterol, Is a Cause of Chronic Disease. Nutrients. 2018; 10(5): 604. https://doi. org/10.3390/nu10050604

30. Bojar I, Wojcik-Fatla A, Owoc A, et al. Polymorphisms of apolipoprotein E gene and cognitive functions of postmenopausal women, measured by battery of computer tests - Central Nervous System Vital Signs. Neuro Endocrinol Lett. 2012; 33(4): 385-92.

31.Bojar I, Owoc J, Wójcik-Fatla A, et al. Cognitive functions, lipid profile, and Apolipoprotein E gene polymorphism in postmenopausal women. Ann Agric Environ Med. 2015; 22(2): 313-9. https://doi. org/10.5604/12321966.1152086

32. Tan BL, Norhaizan ME. Effect of High-Fat Diets on Oxidative Stress, Cellular Inflammatory Response and Cognitive Function. Nutrients. 2019; 11(11): 2579. https://doi.org/10.3390/nu11112579

33. Mergenthaler P, Lindauer U, Dienel GA, et al. Sugar for the brain: the role of glucose in physiological and pathological brain function. Trends Neurosci. 2013; 36(10): 587-597. https://doi.org/10.1016/j. tins.2013.07.001

34.Zanchi D, Meyer-Gerspach AC, Schmidt A, et al. Acute Effects of Glucose and Fructose Administration on the Neural Correlates of Cognitive Functioning in Healthy Subjects: A Pilot Study. Front Psychiatry. 2018; 9: 71. https://doi.org/10.3389/fpsyt.2018.00071

35. Papunen S, Mustakallio-Könönen A, Auvinen J, et al. The association between diabetes and cognitive changes during aging. Scand J Prim Health Care. 2020; 38(3): 281-290. https://doi.org/10.1080/02813432. 2020.1802140

36. Chong CP, Shahar S, Haron $\mathrm{H}$, et al. Habitual sugar intake and cognitive impairment among multi-ethnic Malaysian older adults. Clin Interv Aging. 2019; 14: 1331-1342. https://doi.org/10.2147/CIA.S211534

37. Mortby ME, Janke AL, Anstey KJ, et al. High “Normal” Blood Glucose Is Associated with Decreased Brain Volume and Cognitive Performance in the 60s: The PATH through Life Study. PLoS One. 2013; 8(9): 73697. https://doi.org/10.1371/journal.pone.0073697

38. Qiu Q, Lin X, Sun L, et al. Cognitive decline is related to high blood glucose levels in older Chinese adults with the ApoE $\varepsilon 3 / \varepsilon 3$ genotype. Transl Neurodegener. 2019; 8: 12, https://doi.org/10.1186/s40035-019$0151-2$

39. Moheet A, Mangia S, Seaquist ER. Impact of diabetes on cognitive function and brain structure. Ann N Y Acad Sci. 2015; 1353: 60-71. https://doi.org/10.1111/nyas.12807

40. Kerti L, Witte AV, Winkler A, et al. Higher glucose levels associated with lower memory and reduced hippocampal microstructure. Neurology. 2013; 81(20): 1746-52. https://doi.org/10.1212/01.wnl.0000435561.00234.ee

41. Prasad S, Sajja RK, Naik P, Cucullo L. Diabetes Mellitus and BloodBrain Barrier Dysfunction: An Overview. J Pharmacovigil. 2014; 2(2): 125. https://doi.org/10.4172/2329-6887.1000125

42. Kim B \& Feldman EL. Insulin resistance as a key link for the increased risk of cognitive impairment in the metabolic syndrome. Exp Mol Med. 2015: 47(3): 149. https://doi.org/10.1038/emm.2015.3

43. Gudala K, Bansal D, Schifano F, et al. Diabetes mellitus and risk of dementia: A meta-analysis of prospective observational studies. J Diabetes Investig. 2013; 4(6): 640-650. https://doi.org/10.1111/jdi.12087

44. Zilliox LA, Chadrasekaran K, Kwan JY, et al. Diabetes and Cognitive Impairment. Curr Diab Rep. 2016; 16(9): 87. https://doi.org/10.1007/ s11892-016-0775-x

45. Nazaribadie M, Amini M, Ahmadpanah M, et al. Executive functions and information processing in patients with type 2 diabetes in comparison to pre-diabetic patients. J Diabetes Metab Disord. 2014; 13(1): 27. https://doi.org/10.1186/2251-6581-13-27

46. Kim HG. Cognitive dysfunctions in individuals with diabetes mellitus. Yeungnam Univ J Med. 2019; 36(3): 183-191. https://doi.org/10.12701/ yujm.2019.00255

47. Chudiak A, Uchmanowicz I, Mazur G. Relation between cognitive impairment and treatment adherence in elderly hypertensive patients. Clin Interv Aging. 2018; 13: 1409-1418. https://doi.org/10.2147/CIA. S162701

48. Walker KA, Power MC, Gottesman RF. Defining the Relationship Between Hypertension, Cognitive Decline, and Dementia: a Review. Curr Hypertens Rep. 2017; 19(3): 24. https://doi.org/10.1007/s11906017-0724-3

49. Yuenyongchaiwat K, Baker I, Maratos F, et al. Do Cardiovascular Responses to Active and Passive Coping Tasks predict Future Blood Pressure over a 10-Month Later? Span J Psychol. 2016; 14: 10. https:// doi.org/10.1017/sjp.2016.5

50. Vyas S, Rodrigues AJ, Silva JM, et al. Chronic Stress and Glucocorticoids: From Neuronal Plasticity to Neurodegeneration. Neural Plast. 2016; 2016: 6391686. https://doi.org/10.1155/2016/6391686

51. Li T, Bai Y, Xiang J, et al. Duration of hypertension is associated with cognitive function: a cross-sectional study in Chinese adults. Chin Med J. 2014; 127(11): 2105-10.

52. Uiterwijk R, Huijts M, Staals J, et al. Subjective Cognitive Failures in Patients With Hypertension Are Related to Cognitive Performance 
and Cerebral Microbleeds. Hypertension. 2014: 64(3): 653-7. https:// doi.org/10.1161/HYPERTENSIONAHA.114.03621

53. Fiford CM, Nicholas JM, Biessels GJ, et al. High blood pressure predicts hippocampal atrophy rate in cognitively impaired elders. Alzheimers Dement (Amst). 2020; 12(1): e12035. https://doi.org/10.1002/dad2.12035

54. Shinohara M, Sato N, Shimamura M, et al. Possible modification of Alzheimer's disease by statins in midlife: interactions with genetic and non-genetic risk factors. Front Aging Neurosci. 2014; 23(6): 71. https:// doi.org/10.3389/fnagi.2014.00071

55. Chen ST, Siddarth P, Ercoli LMet al. Modifiable Risk Factors for Alzheimer Disease and Subjective Memory Impairment across Age Groups. PLoS One. 2014; 9(6): e98630. https://doi.org/10.1371/journal. pone.0098630

56. Forte G, De Pascalis V, Favieri F, Casagrande M. Effects of Blood Pressure on Cognitive Performance: A Systematic Review. J Clin Med. 2019; 9(1): 34. https://doi.org/10.3390/jcm9010034

57. Rochlani Y, Pothineni NV, Kovelamudi S, et al. Metabolic syndrome: pathophysiology, management, and modulation by natura compounds. Ther Adv Cardiovasc Dis. 2017; 11(8): 215-225. https:// doi.org/10.1177/1753944717711379

58. Yates KF, Sweat V, Lai YP, et al. Impact of Metabolic Syndrome on Cognition and Brain: A Selected Review of the Literature. Arterioscler Thromb Vasc Biol. 2012; 32(9): 2060-2067. https://doi.org/10.1161/ ATVBAHA.112.252759
59. Cavalieri M, Ropele S, Petrovic K, et al. Metabolic Syndrome, Brain Magnetic Resonance Imaging, and Cognition. Diabetes Care. 2010; 33(12): 2489-2495. https://doi.org/10.2337/dc10-0851

60. Exalto LG, van der Flier WM, van Boheemen CJ, et al. The metabolic syndrome in a memory clinic population: relation with clinical profile and prognosis. J Neurol Sci. 2015; 351(1-2): 18-23. https://doi. org/10.1016/j.jns.2015.02.004

61. Levin BE, Llabre MM, Dong C, et al. Modeling metabolic syndrome and its association with cognition: the Northern Manhattan study. J Int Neuropsychol Soc. 2014: 20(10): 951-60. https://doi.org/10.1017/ S1355617714000861

62. YaffeK, VittinghoffE, PletcherMJ,etal.Earlyadulttomidlifecardiovascular risk factors and cognitive function. Circulation. 2014; 129(15): 1560-7. https://doi.org/10.1161/CIRCULATIONAHA.113.004798

63. Mehra A, Suri V, Kumari S, et al. Association of mild cognitive impairment and metabolic syndrome in patients with hypertension. Asian J Psychiatr. 2020; 30; 53: 102185. https://doi.org/10.1016/j. ajp. 2020.102185

64. Bajerska J, Wozniewicz M, Suwalska A, et al. Eating patterns are associated with cognitive function in the elderly at risk of metabolic syndrome from rural areas. Eur Rev Med Pharmacol Sci. 2014; 18(21): 3234-45. 